\title{
Two dimensional particle swarm optimization algorithm for load flow analysis
}

\author{
Shabana Mehfuz \\ Department of Electrical Engineering, Jamia Millia Islamia \\ New Delhi, 110025, India \\ E-mail:mehfuz_shabana@yahoo.com \\ Sumit Kumar \\ Department of Electrical Engineering, Jamia Millia Islamia \\ New Delhi, 110025, India \\ E-mail: er.sumitkumar21@gmail.com
}

Received 3 July 2013

Accepted 16 July 2014

\begin{abstract}
Methods generally used for load flow analysis have been found to be time consuming and complex. This paper presents an optimization algorithm for load flow analysis inspired from swarm optimization techniques. Particle swarm optimization (PSO) is a type of swarm intelligence that is based on social-psychological principles and provides insights into social behavior, as well as contributes to engineering applications. This algorithm optimizes the power flows on different branches of network using the particles memory based algorithm. The feasibility of the concept has been proved by performing simulations in ETAP environment.
\end{abstract}

Keywords: Particle swarm optimization, load flow analysis, distribution system, power flows. 


\section{Introduction}

Load flow solution is the one of the most commonly used term in power system. Load flow (or power flow) is the solution for the static operating condition of an electric-power transmission system, and is the most frequently performed routine digital-computer power network calculations. Over the last twenty years an enormous amount of effort has been made in research and development on the numerical calculation process Load flow analysis is a very important tool for the analysis of distribution systems and is used in operational as well as planning environments. Real-time applications such as network optimization, switching, state estimation and Var planning in the distribution automation system (DAS) and distribution management system (DMS) work on a robust and effective power flow method ${ }^{1}$. Such a power flow solution must be able to model the special features of distribution systems in sufficient detail. Load flow analysis is the solution of the network under steady state condition subject to certain inequality constraints under which the system operates. Load flow calculation provides the power flows and voltages for a specified power system subject to the regulating capabilities of the generator, condensers and specified net interchange between individual operating system. This information is essential for the continuous monitoring of the present state of the system and analyzing the effectiveness of the alternative plans for the future. The load flow is essential for designing a new power system and for planning extension of existing one for increased load demands control and operation, economic scheduling and exchange of power between the utilities of existing system. One of the most important aspects in power systems nowadays is the voltage stability analysis. Most of the methods used in determining the voltage stability or the maximum loading of the system depend on the solution of the Load flow ${ }^{2}$. It is well known that the problem of load flow is solved using numerical techniques. Previously the gauss-seidal method and Newton-raphson method were used for the load flow analysis. These methods have been found to be problematic in obtaining the load flow solution with the calculation of jacobian matrix. Distribution system load flow analysis is done by conventional forward//backward sweep approach but it takes more time for the convergence ${ }^{3}$. Conventional Newton Raphson method is used to calculate the incremental correction of the state variables in Load Flow where jacobian matrix is used ${ }^{4}$. The well known NewtonRaphson method for the load flow analysis is modified to achieve compatibility for $\mathrm{AC} / \mathrm{DC}$ systems with integrated DC links in the AC network. A modified Jacobian equation comprising of the $\mathrm{DC}$ real and reactive power at the $\mathrm{AC} / \mathrm{DC}$ buses, and their relationship with the $\mathrm{AC}$ system variables has been worked out ${ }^{5,6}$. Artificial Neural Network is used for load flow analysis with real as well as complex numbers ${ }^{7,8}$. A new procedure of load flow which provides solutions for balanced, unbalanced radial distribution network has been proposed which consists of evaluations of a simple algebraic expression of voltage magnitude. Here trigonometric functions are not used and a vector has been used to store the data ${ }^{9}$. Ant Colony algorithm is a new heuristic biological modeling method which has the ability of global searching. An efficient and reliable ant algorithm based load flow algorithm has already been developed $^{10}$. The load flow equations of a power system containing FACTS will be much more nonlinear and the equation set may be non-convex. The conventional NRLF method may not solve these equations satisfactorily $^{17}$. This system was not able to converge when it was loaded near to its maximum point. The aim of the work presented in the paper is the applicability of particle swarm optimization (PSO) and load flow analysis in the management of electrical distribution networks. The term PSO refers to a relatively new family of algorithms that may be used to find optimal or near to optimal solutions to numerical and qualitative problems. PSO can be implemented easily using most of the programming languages since the core of the program can be written in a single line of code and it is both very effective and quick when applied to a diverse set of optimization problems The general objective is to optimize the load flow data for planning and economic scheduling for network management. A new algorithm is developed for the optimization of load flow solution of IEEE 14 bus system in this paper. Particle population used in the algorithm has a memory based updation which is based on their individual and social experiences. Comparison is carried out at every updated 
step. Other advantages of PSO are easy implementation, less number of parameters to be used, less computation time required, efficient global searching, and fast convergence. This paper has been further divided into the following sections. Section 2 describes the characteristics of particle swarm optimization. Section 3 gives the load flow analysis of power system network. Network configuration and simulation is presented in section 4. Paper has been concluded in section 5.

\section{Particle Swarm Optimization}

Particle Swarm Optimization is one of the evolutionary computation techniques. The method has been developed through simulation of simplified social models. A PSO system works on the co-operation between social birds and their adaptation according to the surroundings. This is depicted in figure 1.

A PSO system follows social behavior of the birds and based on social behavior the swarms improve their knowledge and in each iteration they get adapted to that new value. Based on the updated results PSO works as a simulator for different applications. The features of the method are described as follows: This method is based on researches on swarms such as fish schooling and bird flocking. The computation time is short and it requires small amount of memory. It was originally developed for nonlinear optimization problems with continuous variables. However, it can be also used to solve problems with discrete variables. Thereby, it can be applied to mixed integer nonlinear optimization problems with both continuous as well as discrete variables. The above features are suitable for load flow problems as well as for Voltage Control problem because practically efficient methods have not been developed for nonlinear problems with both continuous and discrete variables ${ }^{11}$. The above features allow PSO to handle the load flow problem and also require less computation time. According to the research performed, bird flocking phenomenon, birds find food by flocking (not by each individual). It leads to the assumption that information is shared in flocking. It has been seen from the observations made about behavior of human groups and behavior of each individual i.e. agent, that each individual's behavior is also based on behavior patterns governed by the groups such as customs and other behavior patterns according to the experiences by each individual. This basic assumption is the underlying concept of PSO. PSO is a global optimization algorithm

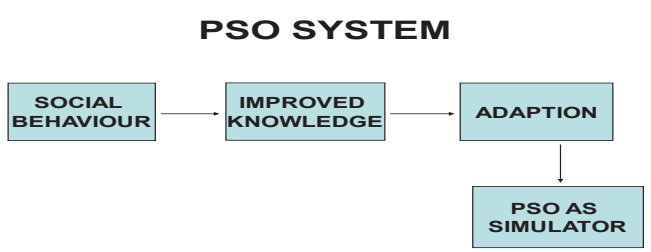

Fig. 1. PSO system steps

for dealing with problems in which a point or surface in an n-dimensional space represents a solution. All of the potential solutions are plotted in this space and provided with an initial velocity ${ }^{27}$. All the particles travel through the solution space, and are evaluated by certain fitness criteria. Over time, particles accelerate toward those with better fitness values. PSO is basically developed through simulation of bird flocking in two dimensional space. The coordinate position of each agent is given its $\mathrm{XY}$ coordinate (fig-2), velocity is expressed as $\mathrm{v}_{\mathrm{x}}$ (the velocity of $\mathrm{X}$-axis) and $\mathrm{v}_{\mathrm{y}}$ (the velocity of $\mathrm{Y}$-axis). The updation of the agent position is made by modifying the position and velocity information. PSO procedures based on the above concept can be described as follows. The bird flocking procedure optimizes a given objective function. Each agent knows its best value so far ( $p_{\text {best }}$ ) and its XY position. Also, each agent has the information of the best value in the group $\left(g_{\text {best }}\right)$ among $p_{\text {bests. }}$ For modifying its position, each agent uses the current velocity and the distance from $p_{\text {best }}$ and $g_{\text {best }}$. The modification can be represented in terms of velocity. Velocity of each agent can be updated by the following equation.

$\mathrm{v}_{\mathrm{i}}=\mathrm{v}_{\mathrm{i}}+\operatorname{rand} *\left(\mathrm{p}_{\text {besti }}-\mathrm{s}_{\mathrm{i}}\right)+$ rand $*\left(\mathrm{~g}_{\text {best }}-\mathrm{s}_{\mathrm{i}}\right)$

where,

$v_{i}$ : velocity of agent $i$,

rand : uniformly distributed random number between 0 and 1 ,

$\mathrm{s}_{\mathrm{i}}$ : current position of agent $\mathrm{i}$,

$\mathrm{p}_{\text {besti }}$ : pbest of agent $\mathrm{i}$,

$\mathrm{g}_{\text {best }}$ : gbest of the group.

Using the above equation, a certain velocity that gradually gets close to $\mathrm{p}_{\text {best }}$ and $\mathrm{g}_{\text {best }}$ can be calculated. The current position (searching point in the solution space) can be modified by the following equation. 
$\mathrm{s}^{\mathrm{k}+1}=\mathrm{s}^{\mathrm{k}}+\mathrm{v}^{\mathrm{k}+1}$

where,

$\mathrm{S}^{\mathrm{k}}$ : current position,

$\mathrm{S}^{\mathrm{k}+1}$ : modified position,

$\mathrm{V}_{\text {orig }}$ : current velocity,

$\mathrm{V}_{\text {mod }}$ : modified velocity,

$\mathrm{V}_{\text {pbest }}$ : velocity based on $\mathrm{p}_{\text {best }}$

$\mathrm{V}_{\text {gbest }}$ : velocity based on $\mathrm{g}_{\text {best }}$

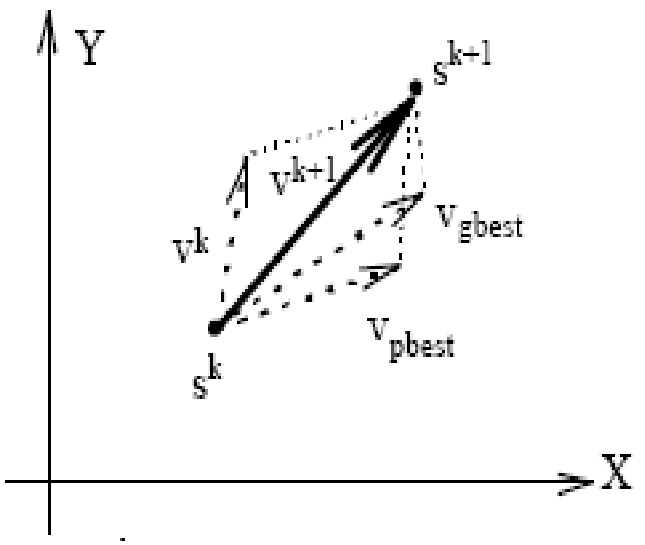

Fig. 2. Searching point in solution space

Fig. 2 presents the concept of updating searching points. PSO makes use of several searching points like Genetic Algorithm (GA) and the searching points gradually get close to the global optimal point using its pbest and gbest. The features of the searching procedure can be summarized as follows:

(i) Initial positions of pbest and gbest are different. By using the different direction of pbest and gbest all agents gradually move towards the global optimum.

(ii) The modified value of the agent position is continuous and the method can be applied to the continuous problem. However, the method can be applied to the discrete problem using grids for $\mathrm{XY}$ position and its velocity.

(iii) There is no inconsistency in searching procedures even if continuous and discrete state variables are utilized with continuous axis and grids for XY positions and velocities. The method can be used for solving mixed integer nonlinear optimization problems with continuous and discrete state variables naturally and easily.

(iv) The above concept is explained using only XY axis (2 dimensional spaces) (Fig. 3). However, the method can be easily applied to n-dimensional problem.

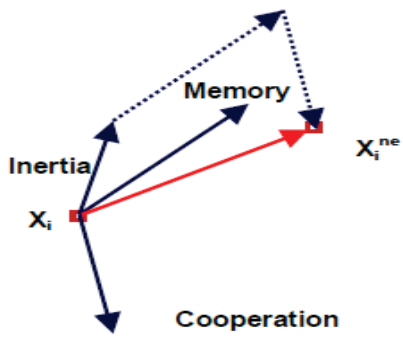

Fig.3. Particle movement components

The particle movement is influenced by the three major components; memory, inertia and co-operation. The movement results in new positions of particle with updated data. The movement of particle start from the current position and it is influenced by the best position of his life as well as the best position found by the other particle in their life ${ }^{22}$.

The topology represents a fully connected graph, and, based on all the statistics, it is concluded that information spreads quickly (fig. 4). Sociologically, it could represent a small and closed community where decisions are taken in consensus. The ring topology represents a regular graph with a minimum number of edges between its nodes. The graph statistics show that information travels slowly along the graph. This allows for different regions of the search space to be explored at the same time, because information of successful regions travels to the other side of the graph in a long time $^{28}$. Graph topology has one particle connected with all the other and hypercube topology has connections of each particle with all the elements in the group. Particle Swarm Optimization is used for other optimization problems in power system too. According to the features of distribution network, more feasible solutions can be produced by updating the rule of position modification in Particle Swarm Optimization for reconfiguration of Distribution Network ${ }^{12}$. At first, PSO algorithm was used to solve optimization problem in continuous space. In order to solve optimization problem in discrete space, Dr. Kennedy and Dr. 
Eberhart introduced PSO algorithm in the form of binary system ${ }^{13}$. Particle Swarm Optimization is also used for optimal steady-state performance analysis of power systems ${ }^{14}$.

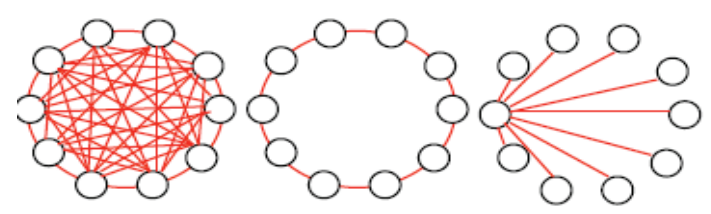

Fig.4. Particle topology in PSO

\section{Load flow analysis}

Load flow analysis is the solution of the network under steady state condition subject to certain inequality constraints under which the system operates. Load flow calculation provides the power flows and voltages for a specified power system subject to the regulating capabilities of the generator, condensers and specified net interchange between individual operating system. This information is essential for the continuous monitoring of the current state of the system and analyzing the effectiveness of the alternative plans for the future. The load flow is necessary because of the reasons given below:

It is essential for designing a new power system and for planning extension of existing one for increased load demands ${ }^{15}$. Load flow is important for economic scheduling, control and operation, planning, and exchange of power between the utilities of existing system. Load flow can determine the best size and as well as the most favorable location for capacitor. Load flow analysis is also required for many other studies such as transient stability, voltage stability and contingency studies ${ }^{16}$. The main objective of load flow is to find the voltage and the angle of the lines during any undesirable condition that exist in the network. Therefore the search for optimized $\mathrm{v}, \delta$ depends upon the two functions of the real and reactive powers of the network. A load flow solution of the power system requires mainly the two steps, formulation of the network equations and suitable mathematical technique for the solution of equations. The load flow equations are simply the power balance equations at each bus, both active and reactive powers. The power balance equation shows that there are no power losses in any bus, as the input power to a bus is equal to the output power from that bus. Load Flow finds the voltage magnitudes and angles of the different system buses that minimize the difference between the input power and the output power from the bus. So the load flow problem can be formulated as an optimization problem $^{17,18}$.

$\mathrm{F}_{\mathrm{pi}}=\Sigma \mathrm{V}_{\mathrm{i}} \mathrm{V}_{\mathrm{j}} \mathrm{Y}_{\mathrm{ij}} \cos \left(\theta_{\mathrm{ij}}+\delta_{\mathrm{i}}-\delta_{\mathrm{j}}\right)-\mathrm{P}_{\mathrm{gi}}+\mathrm{P}_{\mathrm{li}}$

$\mathrm{F}_{\mathrm{qi}}=\Sigma \mathrm{V}_{\mathrm{i}} \mathrm{V}_{\mathrm{j}} \mathrm{Y}_{\mathrm{ij}} \sin \left(\theta_{\mathrm{ij}}+\delta_{\mathrm{i}}-\delta_{\mathrm{j}}\right)-\mathrm{Q}_{\mathrm{gi}}+\mathrm{Q}_{\mathrm{li}}$

These are the load flow equations for active and reactive powers and our optimization problem can be formulated as the minimization of the load flow function.

Minimize $\mathrm{f}(\mathrm{v}, \delta)$

So the objective function of the load flow is to minimize these functions to get the voltages and angles of the buses, which satisfy the power balance equations. As this function is non linear function, we have used particle swarm algorithm to optimize $i^{19}$. At any given load on the system there are two solutions for the load flow (Fig. 5). One is a stable solution (at point 1) and the other one is unstable (at point 2) from the voltage stability point of view. This means that as an optimization problem the Load Flow has two global minima. To overcome this problem we use particle swarm optimization algorithm.

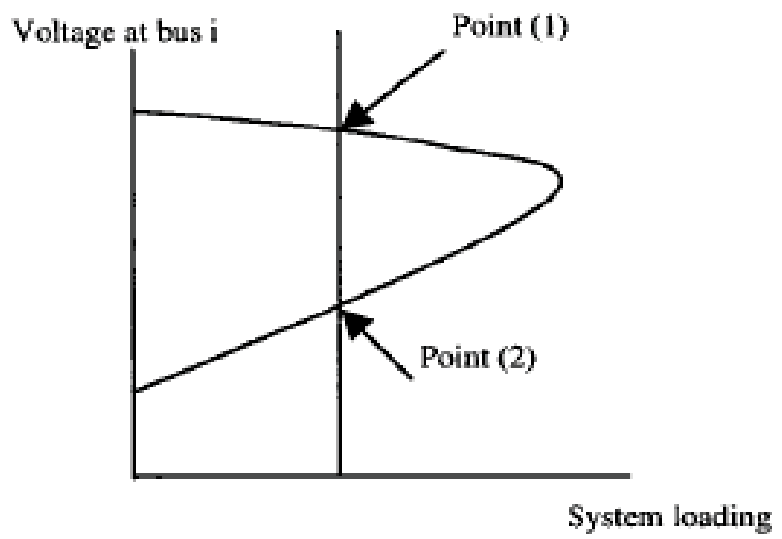

Fig.5. P-V curve for given load

Several algorithms are available for carrying out load flow analysis of power systems. In this paper, we have used three important algorithms - Gauss-Seidel (G-S) Method, Newton-Raphson (N-R) Method, and Fast Decoupled (FDC) method for comparison with the performance of the proposed algorithm. The GaussSeidel method is one of the simplest iterative methods in the solution of non-linear equations. The method is based on the Gauss iterative method and also a flat voltage profile is considered initially ${ }^{17}$. The number of iterations required in this method is more than other methods. On the other hand the time taken per iteration is less than other iterative methods. This method provides better performance for systems with light loading conditions and hence is not recommended for 
heavily loaded systems. N-R method $\operatorname{mon}^{17,18}$ is a very popular and faster solution for load flows analysis. This method requires an initial condition and works well for heavily loaded systems as compared to other methods. FDC method is derived from N-R method. It essentially decouples the effect of reactive power on the angles of complex bus voltage along with the effect of real power on magnitudes of complex bus voltages.

\section{Network configuration and simulation}

IEEE 14- bus system is taken as the network to prove the validity of the particle swarm optimization algorithm for load flow analysis ${ }^{20,21}$. Figure 6 presents the test system which consists of the slack bus (node 1), three PV (nodes 2, 3, and 6), ten PQ buses, and 20 branches. This network has been used in many probabilistic studies. The system of six discrete distributions for the active load (at nodes 3, 6, 9, 10, 11, and 14), four discrete distributions for the reactive load (at nodes 9, 10, 11, and 14), with three to five impulses each and eight normal distributions for active and reactive loads at the remaining buses. The bus voltage is set to $0.44 \mathrm{KV}$. The control variables are transformer tap (t) and reactive compensation (b) at bus 9 .

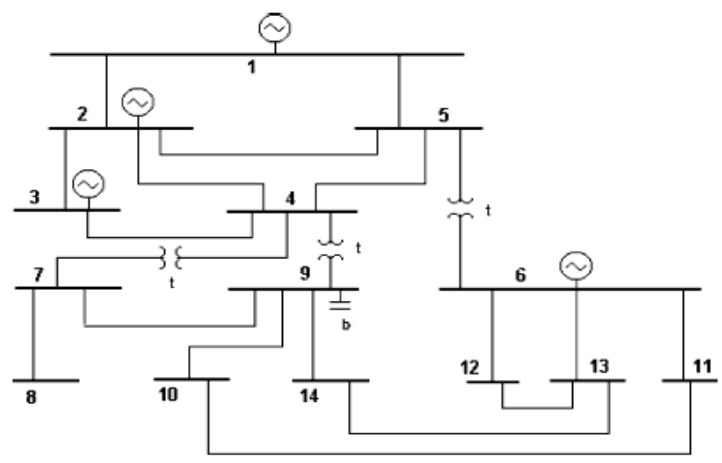

Fig.6. IEEE 14 Bus network

The IEEE 14 bus system is configured in ETAP software (Fig.7) and then the load flow is run to get the voltage magnitude and angles at different buses. This was carried out using Newton Raphson method, GaussSeidel method, and Fast Decoupled method ${ }^{17,18}$. The result is shown in fig. 10 which depicts the voltage profile of all the 14 buses for these methods.

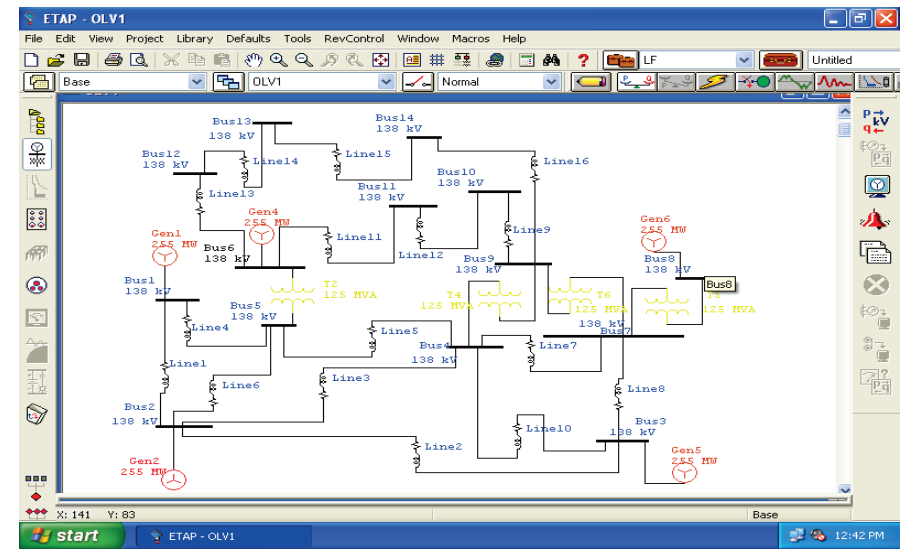

Fig.7. Simulated network in ETAP

The results of load flow analysis of 14 bus network was optimized using particle swarm optimization algorithm $^{25,26}$. This algorithm was used for the optimization of load flow results in MATLAB with PSO toolbox. The tool box screen for the optimization is shown in fig. 8. Two dimensions of search were taken with twenty positions at the starting of search. With each and every iteration, search area reduced and inertia weight also reduced with the reduction in search space. The search process ended, when either number of iterations finished or the search converged. The results of PSO simulations were obtained mostly by convergence of simulation as this is a fast algorithm which takes less computation time also. Since this algorithm converges before the number of iterations are over, it gives results which are very near to the actual bus voltages. The improved results for load flow analysis are presented in graphs given in fig. 9 and fig. 10. It presents a comparison of bus voltages and phase angle. It can seen that voltage of load flow using PSO is much closer to the voltage profile of 14 bus network as compared to Load flow without using PSO algorithm. 


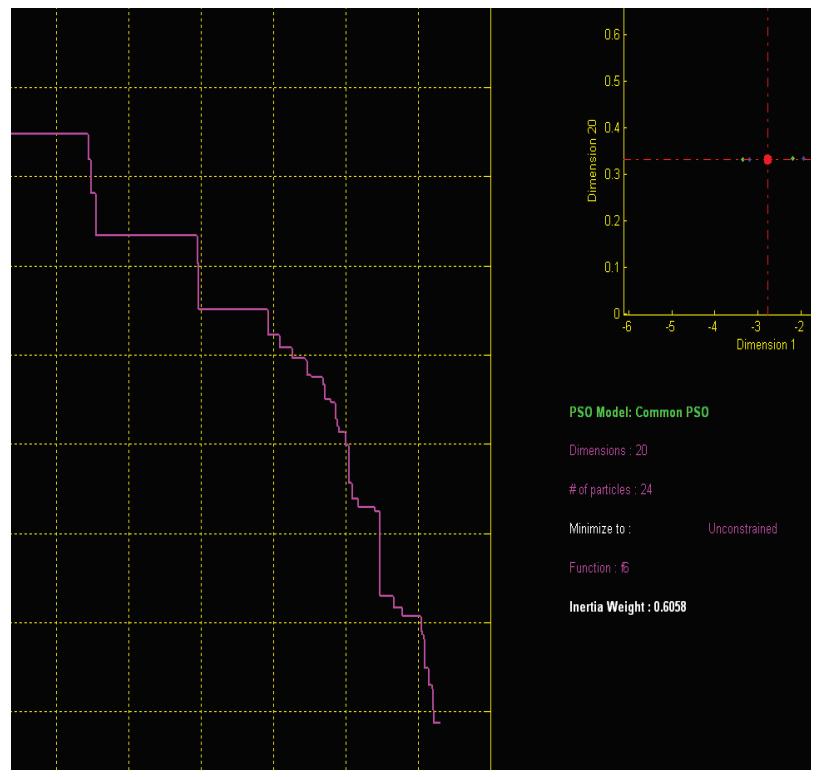

Fig.8. PSO load flow result screen

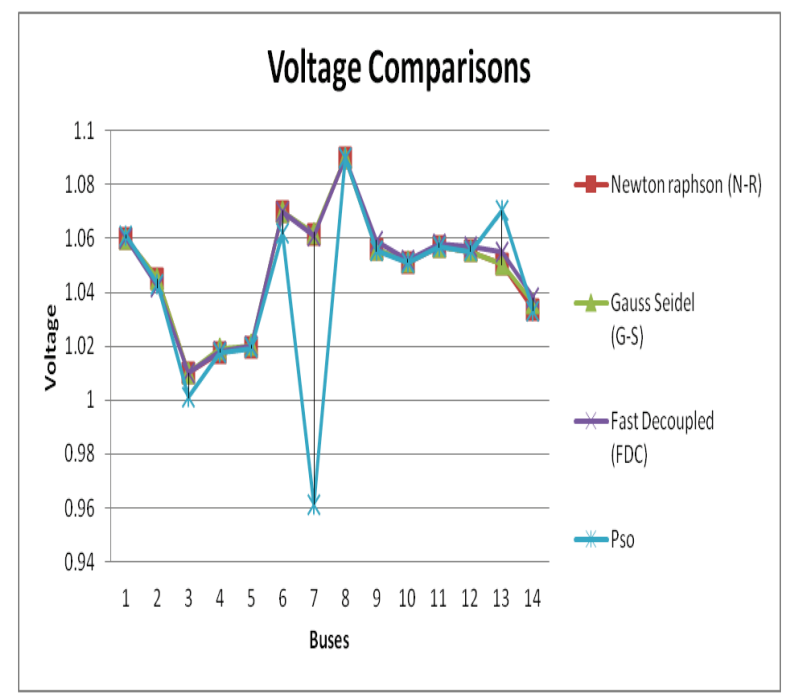

Fig.9. Voltage magnitude comparison

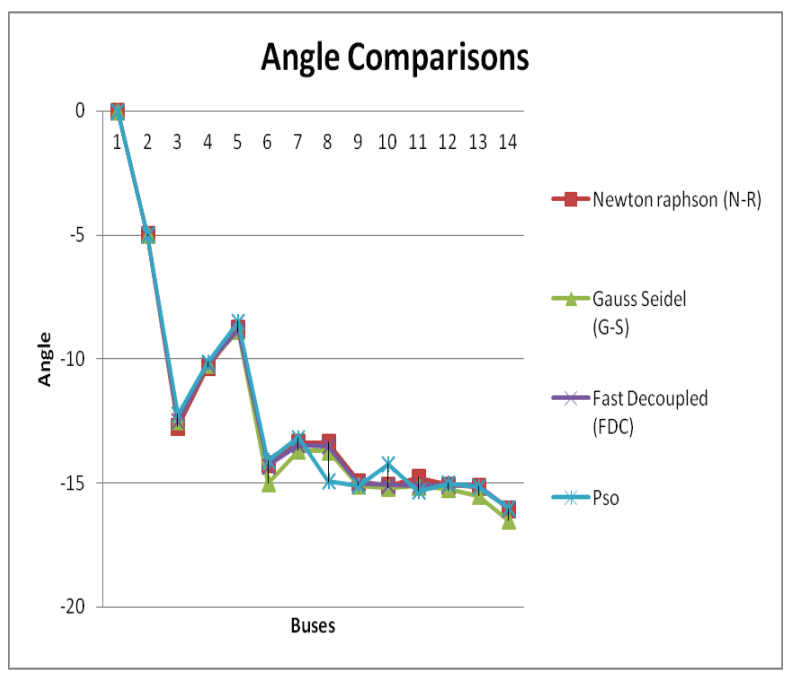

Fig.10. Phase magnitude comparison

This comparison of result is analysed and presented in Table 1 for all the buses and their respective voltages and angles. Standard deviation has been calculated and it shows the closeness of the results with the actual values. The bus voltages at bus 4 is same as standard deviation is zero for this one. For other buses also the standard deviation is quite low. The angle is different for each and every bus except bus 1 .

Table 1. Comparison of result (Voltage)

\begin{tabular}{|r|r|r|r|r|r|}
\hline $\begin{array}{l}\text { Bus } \\
\text { No }\end{array}$ & $\begin{array}{l}\text { Newton } \\
\text { raphson } \\
\text { (N-R) }\end{array}$ & $\begin{array}{l}\text { Gauss } \\
\text { Seidel } \\
\text { (G-S) }\end{array}$ & $\begin{array}{l}\text { Fast } \\
\text { Decoupled } \\
\text { (FDC) }\end{array}$ & PSO & $\begin{array}{l}\text { Standard } \\
\text { Deviation }\end{array}$ \\
\hline 1 & 1.06 & 1.06 & 1.06 & 1.061 & 0.0005000 \\
\hline 2 & 1.045 & 1.045 & 1.042 & 1.043 & 0.0015000 \\
\hline 3 & 1.01 & 1.01 & 1.01 & 1.001 & 0.0045000 \\
\hline 4 & 1.0177 & 1.019 & 1.018 & 1.018 & 0.0006164 \\
\hline 5 & 1.0195 & 1.020 & 1.02 & 1.019 & 0.0004573 \\
\hline 6 & 1.07 & 1.07 & 1.07 & 1.062 & 0.0040500 \\
\hline 7 & 1.0615 & 1.062 & 1.061 & 0.961 & 0.0501016 \\
\hline 8 & 1.09 & 1.09 & 1.09 & 1.09 & 0 \\
\hline 9 & 1.0559 & 1.056 & 1.059 & 1.055 & 0.0016378 \\
\hline 10 & 1.051 & 1.051 & 1.052 & 1.051 & 0.0004717 \\
\hline 11 & 1.0569 & 1.057 & 1.058 & 1.057 & 0.0005354 \\
\hline 12 & 1.0552 & 1.055 & 1.057 & 1.055 & 0.0009000 \\
\hline 13 & 1.0504 & 1.050 & 1.055 & 1.070 & 0.0094845 \\
\hline 14 & 1.0335 & 1.036 & 1.038 & 1.033 & 0.0022411 \\
\hline
\end{tabular}


Table 2. Comparison of result (Angle)

\begin{tabular}{|r|l|r|r|r|r|}
\hline $\begin{array}{l}\text { Bus } \\
\text { No }\end{array}$ & $\begin{array}{l}\text { Newton } \\
\text { raphson } \\
\text { (N-R) }\end{array}$ & $\begin{array}{l}\text { Gauss } \\
\text { Seidel } \\
\text { G-S })\end{array}$ & $\begin{array}{l}\text { Fast } \\
\text { Decouple } \\
\text { d } \\
\text { (FDC) }\end{array}$ & PSO & $\begin{array}{l}\text { Standard } \\
\text { Deviation }\end{array}$ \\
\hline 1 & 0 & 0 & 0 & 0 & 0 \\
\hline 2 & -4.9847 & -5 & -5 & -4.9815 & 0.009844 \\
\hline 3 & -12.723 & -12.5 & -12.5 & -12.223 & 0.204719 \\
\hline 4 & -10.311 & -10.21 & -10.2 & -10.141 & 0.070571 \\
\hline 5 & -8.7718 & -8.87 & -8.8 & -8.5013 & 0.161676 \\
\hline 6 & -14.216 & -15 & -14.3 & -14.1 & 0.405706 \\
\hline 7 & -13.356 & -13.68 & -13.45 & -13.16 & 0.215953 \\
\hline 8 & -13.361 & -13.71 & -13.5 & -14.931 & 0.718141 \\
\hline 9 & -14.937 & -15.1 & -15 & -15.097 & 0.079349 \\
\hline 10 & -15.097 & -15.2 & -15.08 & -14.236 & 0.447982 \\
\hline 11 & -14.788 & -15.13 & -15.1 & -15.321 & 0.220722 \\
\hline 12 & -15.075 & -15.25 & -15.07 & -15.012 & 0.102886 \\
\hline 13 & -15.156 & -15.5 & -15.12 & -15.152 & 0.179392 \\
\hline 14 & -16.031 & -16.5 & -16.04 & -16.025 & 0.234081 \\
\hline
\end{tabular}

\section{Conclusion}

The objective of this work was to use the concept of particle swarm optimization for the non linear load flow optimization. PSO has been used for power system management. The theoretical framework chosen was based on the search of swarms for food. Individuals gain evolutionary advantage by sharing information among a group when a task is given to them. This idea leads to the search of solution in to an optimization problem. The initial aim of the particle swarm concept was to graphically simulate the graceful and unpredictable choreography of a bird flock, the motive of discovering patterns that govern the ability of birds to fly synchronously, and to suddenly modify direction with a regrouping in an optimal fashion. From the initial objective, the concept evolved into a simple and efficient optimization algorithm as the voltages and angles obtained by Load Flow using PSO are much closer than the ideal values.

\section{References}

1. Jen- Hao- Tang, A network topology based three phase load flow for distribution system, Proceedings of Natural Science Council ROC(A) 24(4) (2000) 259-264.

2. A. M. El-Zonkoly, Optimal Sizing of SSSC Controllers to Minimize Transmission Loss and a Novel Model of SSSC to Study Transient Response, World Academy of Science,
Engineering and Technology (24) (2008) 825-831.

3. G. W. Chang, S. Y. Chu, and H. L. Wang, A Simplified Forward and Backward Sweep Approach for Distribution System Load Flow Analysis, IEEE International Conference on Power System Technology (Dec. 2006), pp. 990-997.

4. K.B.Porate, M.J.Katira, Load Flow Analysis of 132 / 11 $\mathrm{kV}$ Distribution Sub Station using Static Var Compensator for Voltage Enhancement - A Case Study, Tencon, IEEE region 10 conference (2009), pp. 1-5.

5. Youssef A. Mobarak, Modified Load Flow Analysis for Integrated AC/DC Power Systems, IEEE Power System Conference MEPCON vol 2 (2008), pp. 402-405.

6. Panosyan, B. R. Oswald, Modified Newton raphson load Flow analysis for integrated AC/DC Power Syatem, IEEE universities power engineering conference, UPEC, vol 3, (2004), pp. 1223-122 .

7. W.L.Chan,A.T.P.So and L.L.Lai, Initial applications of complex artificial neural networks to load-f low analysis, IEEE proc. generation transmission distribution, 147(6), (November 2000), pp.361-366.

8. Amit Jain, S. C. Tripathy, R. Balasubramanian, Kamal Garg and Yoshyuki Kawazoe, Neural Network Based Stochastic Load Flow Analysis, IEEE International Conference on Power System Technology - POWERCON (2004), pp. 1845-1850.

9. C. Lakshminarayana and M.R. Mohan, An Improved Load Flow Method for the Analysis of Pre / Post Fault Distribution Systems, IEEE Proceedings of India International Conference on Power Electronics (2006), pp. 239-246.

10. Y. Kumar, K. Dwivedi, Ganga Agnihotri, Development of ANT Algorithm For Load Flow Analysis, IEEE Power system conference and exposition, PSCE -09,PES (2009), pp. 451-458.

11. Vladimiro Miranda and Nuno Fonseca, Evolutionary Particle Swarm Algorithm (EPSO) Applied to Voltage var control, IEEE/PES, Transmission and Distribution conference and exhibition,Asia Pacific, vol 2 (2002), pp. 745-750.

12. Lin Lu, Qi Luo, Jun-yong Liu, Chuan Long, An Improved Particle Swarm Optimization for reconfiguration of Distribution Network, IEEE Fourth International Conference on Natural Computation, vol.3 (2008), pp. 453-457.

13. J Kennedy, R Eberhart, A Discrete binary version of the particle swarm algorithm, Proceedings of IEEE International Conference on system, Man and Cybernetics, Vol 5 (1997), pp. 4104-4108.

14. John G. Vlachogiannis and Kwang Y. Lee, A Comparative Study on Particle Swarm Optimization for Optimal Steady-State Performance of Power Systems, IEEE trans. on power system, 21(4) (Nov. 2006) 1718-1728. 
15. A.EL-Dib, Hosam, Metwalli, Load flow using hybrid particle swarm optimization, IEEE conference on power systems application, vol 3 (2004), pp. 1-7.

16. T. S. Karakatsanis and N. D. Hatziargyriou, Probabilistic constrained load flow based on sensitivity analysis, IEEE Trans. Power Syst. 9(4) (1994), 1853-1860.

17. Glem W. Stagg, Ahmed H. Alabiad, Computer methods in Power system analysis, (Macgraw-Hill, 1968).

18. Weimin Ma and James s.Thorp, An efficient algorithm to locate all the load flow solution, IEEE Trans. on power system, 8(3) (Aug 1993), 1077-1084.

19. Xiaodong Yin, Application of genetic algorithm to multiple load flow solution problem in electrical power system, IEEE proceedings of the 32nd conference on decision and control (December 1993), pp. 345-353.

20. H. Yoshida, K. Kawata, Y. Fukuyama, S. Takayama, and Y.Nakanishi, A practical swarm optimization for reactive power and voltage control considering voltage security assessment, IEEE Trans. Power Syst., 15(4) (Nov. 2000) 1232-1239.

21. Kennedy J and Eberhart R.C, Particle swarm optimization, Proceedings of the IEEE International Conference on Neural Networks, (4) (1995), pp.1942-1948.

22. J. Kennedy, The particle swarm: Social adaptation of knowledge, Proc. IEEE Int. Conf. Evol. Comput. Indianapolis (1997), pp. 303-308.

23. M. Clerc and J. Kennedy, The Particle Swarm: Explosion, Stability, and Convergence in a Multi-Dimensional Complex Space, IEEE Trans. on Evolutionary Computation, 6(1) (2002) 58-73.

24. Shi, Y., Eberhart, R.C., Parameter Selection in Particle Swarm Optimization, IEEE Proceedings of the Seventh Annual Conference on Evolutionary Programming, (1998).

25. Joseph, John, Kwanty, and Moshe, Supervisory control of electric power transmission network, IEEE Trans .power syst, 10(2) (May 1995), 389-398.

26. Jang-Ho Seo1, Chang-Hwan Im2, Chang-Geun Heo3, JaeKwang Kim1, Hyun-Kyo Jung1, and Cheol-Gyun Lee, Multimodal Function Optimization Based on Particle Swarm Optimization, IEEE Transactions on magnetics, 42(4) (April 2006) 856-863.

27. Michael G. Hinchey, Roy Steritt, Chris Rouff, Swarms \& Swarms Intelligence, Journal of Computers, 40(4) (April 2007) 111-113.

28. S.N.Sivanandam, P.Visalakshi, Multiprocessor Scheduling Using Hybrid Particle Swarm Optimization with Dynamically Varying Inertia, International Journal of Computer Science \& Applications 4(3) (2007) 95-106. 